\title{
Lettre à la rédaction : La cocaïne n'en était pas : une soumission chimique originale
}

\author{
Letter to the editor: \\ It was not cocaine: an original drug \\ facilitated crime
}

Véronique DUMESTRE-TOULET ${ }^{(1) *}$, Alain EYQUEM(1), Marion VILLAIN ${ }^{(2)}$, Pascal KINTZ ${ }^{(2)}$

(1) Laboratoire Toxgen, 11, rue du Commandant Cousteau, 33100 Bordeaux (2) Laboratoire ChemTox, 3, rue Grüninger , 67400 Illkirch

*Auteur à qui adresser la correspondance : Véronique DUMESTRE-TOULET, Laboratoire Toxgen, 11, rue du Commandant Cousteau, 33100 Bordeaux -E-mail : vdumestr@toxgen.fr

(Reçu le 25 août 2006 ; accepté après modification le 5 mars 2007)

\section{Introduction}

La soumission chimique est l'administration de substances psychoactives à l'insu d'une personne à des fins délictueuses ou criminelles. Le but recherché par l'agresseur est une diminution de la vigilance, de la conscience et des facultés de jugement et de résistance du sujet. Les produits utilisés sont le plus souvent actifs à faibles doses, agissent rapidement et sont très vite éliminés de l'organisme, d'où la difficulté de leur mise en évidence. Ils provoquent en outre, une amnésie antérograde au moment des faits et donc des dépôts de plaintes et des prélèvements tardifs (délai moyen des plaintes en France : $17 \mathrm{~h}$ ). L'analyse des cheveux, augmentant la fenêtre de détection des xénobiotiques, fournit alors de précieux résultats pour caractériser les faits.

\section{Cas clinique}

Le cas présenté rapporte l'agression sexuelle de deux adolescents après une soumission plutôt originale, de part la voie d'administration du produit psychoactif.

Deux adolescents de 15 et 16 ans, sous tutelle, acceptent de suivre à son domicile un individu qui leur propose une ligne de cocaïne. Ils sniffent effectivement une poudre blanche à l'aide d'un billet de 1 dollar et absorbent alcool et jus de fruits. Ils s'endorment rapidement et se réveillent le lendemain, nus, dans le lit de l'individu, sans souvenirs de la nuit écoulée. Une plainte sera déposée 4 jours après par les familles d'accueil. Lors de la perquisition au domicile du suspect est saisi un billet vert ainsi que diverses boissons.

Une expertise toxicologique avec analyse de sang, urines, 
cheveux des deux garçons et du billet de banque est requise pour mettre en évidence les éventuelles substances en présence. Les prélèvements de sang et urines sont effectués 5 jours après les faits alors que les prélèvements de cheveux (une mèche orientée d'environ 100 cheveux prélevée en occipital pour chaque sujet) sont effectués 4 semaines après les faits selon le protocole usuel.

\section{Matériel et méthodes}

Les fluides biologiques ont été analysés selon les procédures habituelles du laboratoire : L'éthanol est mesuré par GC/FID, les stupéfiants (cannabinoïdes, opiacés, cocaïniques, amphétaminiques, LSD, alcaloïdes du datura) et les médicaments psychoactifs (benzodiazépines, zolpidem, zopiclone, phénothiazines, barbituriques, carbamates, GHB) sont recherchés par technique ELISA sur microplaques et trousses CEDIA sur automate et/ou par techniques LC/DAD et GC/MS.

L'identification et le dosage de molécules précitées et en particulier de benzodiazépines dans le sang et les urines sont effectués après extraction liquide/liquide à pH 10 par un mélange dichlorométhane/éther diéthylique $(90 / 10, \mathrm{v} / \mathrm{v}, 5 \mathrm{ml})$ et analyse en chromatographie liquide couplée à la spectrométrie de masse en tandem (LC-MS/MS) sur un système 2695 Alliance Waters couplé à un détecteur Quattro micro triple quadrupole (Micromass/Waters).
L'échantillon de cheveux de chaque sujet est décontaminé/coupé au ciseaux en fragments d' $1 \mathrm{~mm}$, incubé avec $1 \mathrm{ml}$ de $\mathrm{NaOH} 0,1 \mathrm{~N}$ à $95^{\circ} \mathrm{C}$ pendant $15 \mathrm{~min}$ et extrait par le même mélange de solvants en présence de diazepam- $\mathrm{d}_{5}$ et 7 aminoclonazépam- $\mathrm{d}_{4}$ comme étalons internes puis analysé par LC-MS/MS selon la technique développée par Villain et coll. (1).

\section{Résultats}

Les analyses effectuées ne mettent pas en évidence la présence d'éthanol et de stupéfiants, autre que le cannabis. La cocaïne n'est pas identifiée dans aucun des milieux biologiques, ni sur le billet de banque.

Les analyses réalisées dans le sang, les urines et les cheveux révèlent la présence de benzodiazépines. Le clonazépam est identifié sur le billet d' 1 dollar examiné. Le 7 aminoclonazépam est identifié 5 jours après l'exposition présumée, dans le sang des 2 sujets à une concentration respectivement de 7 et $10 \mu \mathrm{g} / \mathrm{L}$ et dans les urines à des concentrations de 58 et $233 \mu \mathrm{g} / \mathrm{L}$. Les analyses réalisées dans les cheveux mettent en évidence le 7 aminoclonazépam à des concentrations respectivement de 141 et $336 \mathrm{pg} / \mathrm{mg}$ dans un segment $0-2$ ou $0-3 \mathrm{~cm}$ pour chaque sujet.

Le chromatogramme obtenu lors de l'analyse d'un extrait sanguin du sujet 1 est représenté figure 1. Le chromatogramme obtenu lors de l'analyse d'un extrait de cheveux (segment $0-2 \mathrm{~cm}$ ) du sujet 1 est représenté figure 2.

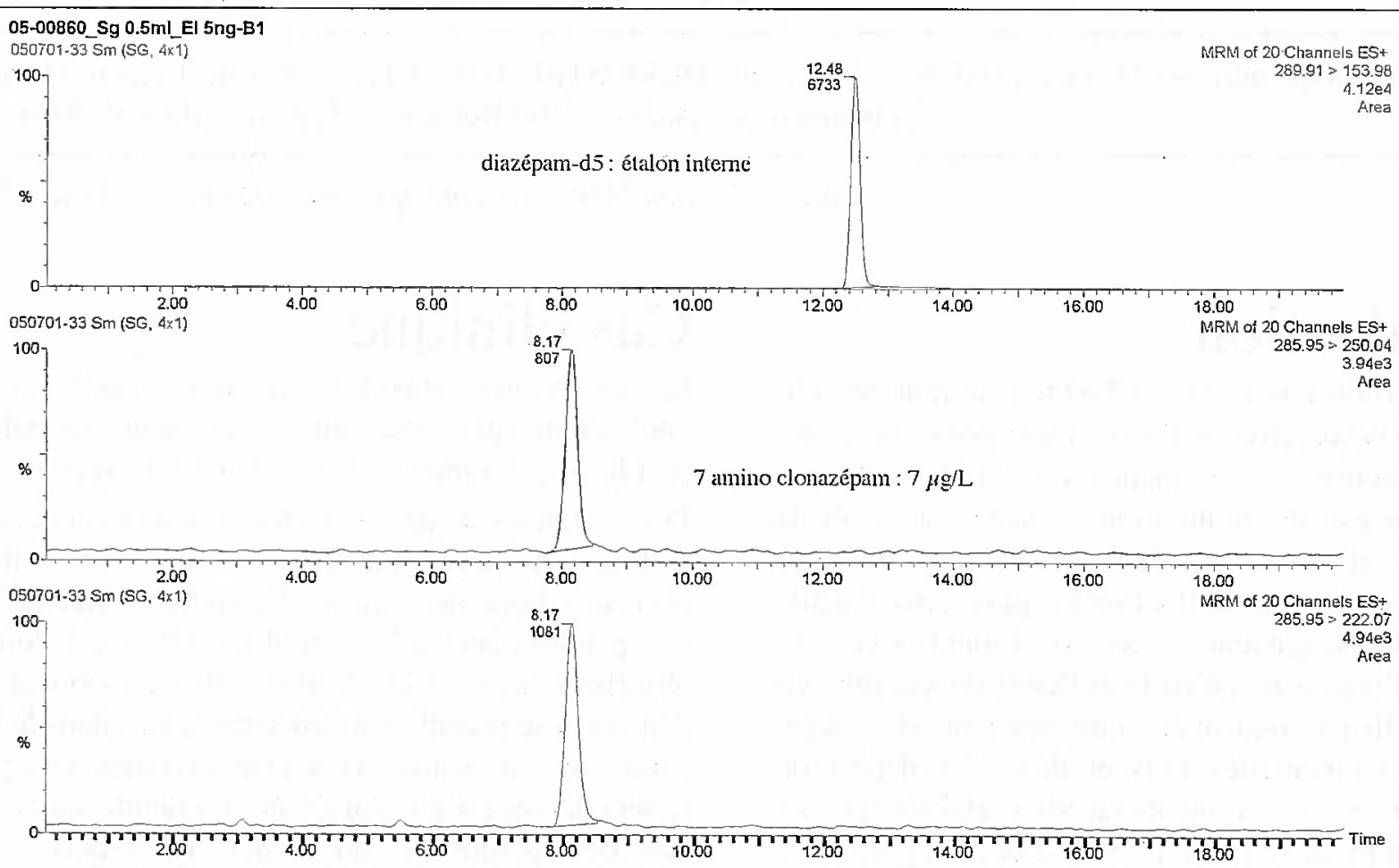

Figure 1 : Chromatogramne d'un extrait sanguin du sujet 1 représentant de haut en bas, l'étalon interne (diazépam-d5) et le 7 aminoclonazépan (2 transitions). 


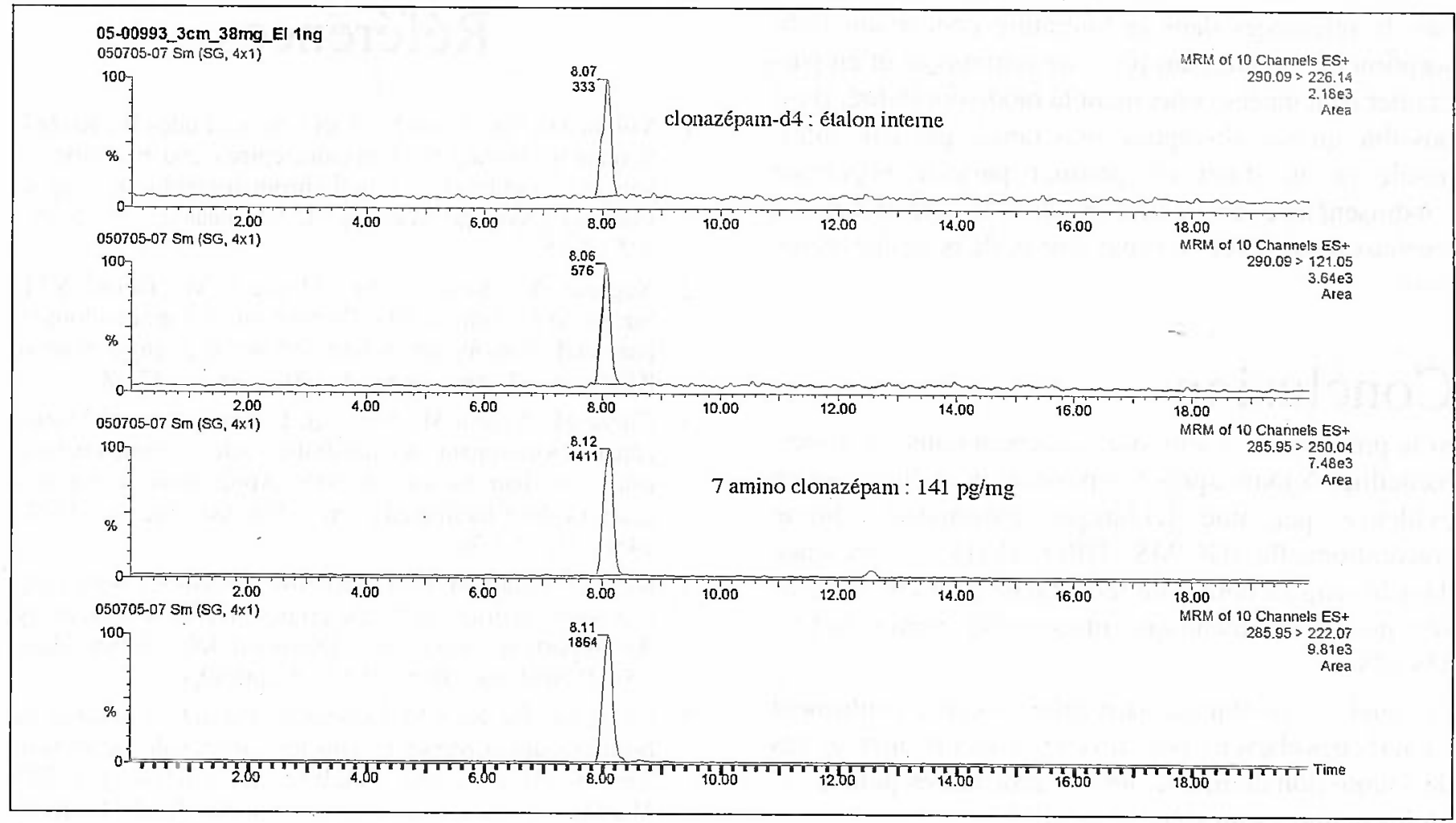

Figure 2 : Chromatogranme d'un extrait de cheveux $(3 \mathrm{~cm})$ du sujet 1 représentant de haut en bas, 2 transitions de l'étalon. interne (clonazépam-d4) et 2 transitions du 7 aminoclonazépam.

\section{Discussion}

Le clonazépam n'est pas identifié dans les échantillons biologiques, fluides et cheveux. La molécule, comme les autres nitrobenzodiazépines, est très instable et se dégrade très rapidement in vitro. Par ailleurs, la molécule est hydrolysée par une réaction de biotransformation hépatique dans l'organisme en 7 aminoclonazépam, métabolite actif, que l'on retrouve dans les fluides biologiques et les cheveux (2).

Le clonazépam (Rivotril $($ ) $)$ est une molécule de la famille des benzodiazépines prescrite pour ses propriétés anticonvulsivantes, sédatives, anxiolytiques et myorelaxantes, à demi-vie d'élimination relativement longue (19 à 60 h). Les effets secondaires, recherchés par un éventuel agresseur, sont des sensations ébrieuses, une hypotonie musculaire, une baisse de vigilance et une amnésie antérograde. Le Rivotril@ est souvent retrouvé dans les affaires de soumission chimique sans doute à cause de la facilité d'administration du produit sous la forme goutte, dont l'excipient est sucré et fruité.

Les résultats observés dans cette expertise sont en faveur d'un cas de soumission chimique par le Rivotril ${ }^{\circledR}$, car l'étude des dossiers médicaux des 2 sujets montraient que ni l'un ni l'autre n'étaient traités pour épilepsie.
La caractérisation d'une exposition à une prise unique de Rivotril@ peut-être réalisée aujourd'hui à partir d'une analyse de cheveux par technique LC-MS/MS $(2,3)$. Plusieurs cas de soumission chimique avec du clonazépam sont cités dans la littérature : Cheze et coll.(3) décrivent une concentration de $22 \mathrm{pg} / \mathrm{mg}$ de 7 aminoclonazépam dans les cheveux d'un volontaire sain après la prise d'un comprimé de Rivotril@ $2 \mathrm{mg}$ ainsi qu'une concentration de $3,2 \mathrm{pg} / \mathrm{mg}$ dans les poils axillaires d'un sujet victime d'une soumission chimique 4 mois auparavant, Kintz et coll. (4) décrivent la cas d'une jeune fille abusée sexuellement par 3 hommes sans pouvoir opposer de résistance et chez qui l'analyse d'un segment de cheveux correspondant à la période des faits révèle la présence de 7 aminoclonazépam (135 pg/mg). Cheze et coll. (5) ont établi à partir de cas publiés et de cas du laboratoire, une corrélation entre les quantités de clonazépam absorbées et les concentrations mesurées dans les cheveux (2 à $371 \mathrm{pg} / \mathrm{mg}$ ) et indiquent que ces concentrations varient selon la quantité absorbée et le caractère chronique de l'exposition.

Les concentrations mesurées dans les cheveux des deux sujets sont compatibles avec une exposition récente. Il est cependant difficile de préciser si cette exposition est importante malgré les concentrations élevées mesurées (141 et 336 pg/mg). En effet, il n' y a 
pas de références dans la littérature concernant l'absorption du clonazépam par voie intranasale et en particulier de données concernant la biodisponibilité. Il est possible qu'une absorption plus rapide par voie intranasale et un shunt du premier passage hépatique conduisent à des concentrations dans le sang et dans les cheveux plus élevées que par voie orale pour une même dose.

\section{Conclusion}

Si la présence de 7 amino-clonazépam dans les urines, recueillies 5 jours après l'exposition peut être mise en évidence par une technique chromatographique conventionnelle (GC/MS, HPLC/DAD), la présence dans le sang au bout d'un tel délai ne peut être objectivée que par une technique ultrasensible comme la LCMS/MS

Les analyses pratiquées dans cette expertise confirment l'intérêt des cheveux pour révéler et documenter les cas de soumission chimique, lors de procédures judiciaires où les plaintes sont souvent tardives.

Malgré la publication récente de donnéés dans la littérature à propos de la relation dose-concentration de clonazépam dans les cheveux (5), il n'est pas possible de préciser l'importance de l'exposition des 2 sujets de cette expertise car il n'y a pas de données concernant l'administration intranasale de Rivotril@.

\section{Références}

1. Villain M., Concheiro M., Cirimele V., Ludes B., Kintz P. Screening method for benzodiazepines and hypnotics in hair at pg/mg level by liquid chromatography-mass spectrometry/mass spectrometry. J. Chromatogr. B. 2005 ; $825: 72-8$.

2. Negrusz A., Bowen A.M., Moore C.M., Dourd S.M., Strong M.F., Janicak P.G. Deposition of 7-aminoclonazepam and clonazepam in hair following a single dose of Klonopin. - J. Anal. Toxicol. 2002 ; 26(7) : 471-8.

3. Cheze M., Villain M., Pépin G. Determination of bromazepam, clonazepam and metabolites after a single intake in urine and hair by LC-MS/MS. Application to forensic cases of drug facilitated crimes. Forensic Sci. Int. 2004 ; 145(2-3) : 123-30.

4. Kintz P., Villain M. Hair in forensic toxicology with a special focus on drug-facilitated crimes, in Hair in toxicology. An important bio-monitor. Desmond John Tobin editor, RSC Publishing, 2005, 89-103, Cambridge.

5. Cheze M., Deveaux M. Lenoan A., Pépin G. Clonazépam, bromazépam et zolpidem dans les cheveux de victimes de soumission chimique: analyse quantitative par LCMS/MS et relation dose-concentration. Anal. Toxicol . Anal. $2005 ; 17(4)$ : 269-73. 